\title{
ARTICLE OPEN \\ Localized corrosion of low-carbon steel at the nanoscale
}

Steven C. Hayden ${ }^{1}$, Claire Chisholm ${ }^{2,7}$, Rachael O. Grudt $\mathbb{D}^{1}$, Jeffery A. Aguiar $\mathbb{D}^{3}$, William M. Mook ${ }^{2}$, Paul G. Kotula $^{4}$, Tatiana S. Pilyugina ${ }^{1}$, Daniel C. Bufford ${ }^{5}$, Khalid Hattar ${ }^{2}$, Timothy J. Kucharski (D) ${ }^{1}$, Ihsan M. Taie ${ }^{6}$, Michele L. Ostraat ${ }^{1}$ and Katherine L. Jungjohann ${ }^{2}$

Mitigating corrosion remains a daunting challenge due to localized, nanoscale corrosion events that are poorly understood but are known to cause unpredictable variations in material longevity. Here, the most recent advances in liquid-cell transmission electron microscopy were employed to capture the advent of localized aqueous corrosion in carbon steel at the nanoscale and in real time. Localized corrosion initiated at a triple junction formed by a solitary cementite grain and two ferrite grains and then continued at the electrochemically-active boundary between these two phases. With this analysis, we identified facetted pitting at the phase boundary, uniform corrosion rates from the steel surface, and data that suggest that a re-initiating galvanic corrosion mechanism is possible in this environment. These observations represent an important step toward atomically defining nanoscale corrosion mechanisms, enabling the informed development of next-generation inhibition technologies and the improvement of corrosion predictive models.

npj Materials Degradation (2019)3:17; https://doi.org/10.1038/s41529-019-0078-1

\section{INTRODUCTION}

The ubiquity of steel in infrastructure makes aqueous steel corrosion a global concern, with negative repercussions impacting most industrial sectors. ${ }^{1}$ In the oil and gas industry, pipeline failures and the resulting disasters are avoided primarily via strategic replacement programs, which rely on predictive models to develop appropriate replacement timelines. ${ }^{2}$ These predictive models have been continually updated since their initial development in the early $1970 \mathrm{~s}^{3}{ }^{3}$ however, even the models employed today often rely on semi-empirical correction factors derived from field observations or bulk-scale experiments to fill gaps in basic scientific understanding. ${ }^{4}$ As a result, these predictive models can fail in practice, with repercussions of failure ranging from mild to catastrophic. $^{5}$

Despite longstanding research efforts, steel corrosion continues to present challenges, due in large part to its intrinsic complexity. Myriad self-compounding environmental factors can completely alter the mechanisms by which corrosion progresses, even when only one factor is changed. This complexity presents a formidable problem for the scientific community to approach, resulting in gaps in the mechanistic understanding of how corrosion progresses in certain environments. Recent studies have suggested that nanoscale processes at heterogeneous sites in materials are a likely culprit for deviation between predictive models and observed corrosion rates from the field. ${ }^{6}$ A timedependent understanding of accelerated nanoscale processes in steel corrosion under aqueous flow will help to narrow this knowledge gap.

Two known classes of concurrent mechanisms govern mild carbon steel corrosion in wet environments-uniform and localized corrosion. Uniform corrosion processes in low-carbon steel are well accounted for on the bulk scale, where localized corrosion events are generally incorporated into empirical corrosion rates. Uniform aqueous corrosion involves non-specific material loss via solvation of oxidized iron at the surface of the steel. ${ }^{7}$ On the other hand, localized corrosion results when discrete regions of the metal surface are selectively attacked by the corrosive medium, resulting in accelerated corrosion rates in specific regions relative to the bulk surface. With the tendency to penetrate the steel surface in a relatively short period of time, localized corrosion is difficult to predict or detect, making it the cause of most corrosion-related material failures. ${ }^{8}$ The two mechanisms work in concert; as localized corrosion exposes a greater surface area to the corrosive medium, the damage caused by uniform corrosion is also exacerbated by the increased exposed surface area.

Pitting in steel initiates at or near inclusions within the microstructure, such as MnS and iron carbides. ${ }^{9,10}$ However, it is not known why some inclusions, even of the same composition, are more electrochemically active than others. ${ }^{11}$ There are three theories explaining the seemingly stochastic mechanism behind inclusion-induced pitting: (1) iron matrix surface orientation, ${ }^{12}(2)$ galvanic mediation, ${ }^{11}$ or (3) a disordered and strained iron matrix. $^{13,14}$ Iron carbide inclusions, specifically cementite, are of particular interest in the study of carbon steel corrosion. These cementite inclusions are a common microstructural feature of mild steels, and cementite is cathodic to ferrite, ${ }^{15}$ leading to preferential etching of ferrite when it is in contact with both cementite and with an electrolytic medium (e.g., $\mathrm{H}_{2} \mathrm{O}$ ). A better understanding of the mechanisms by which corrosion initiates and progresses at these types of interfaces in steel will be key to mitigating corrosion-related losses.

\footnotetext{
'Aramco Services Company, Aramco Research Center, Boston, MA 02139, USA; ${ }^{2}$ Sandia National Laboratories, Center for Integrated Nanotechnologies, Albuquerque, NM 87185 , USA; ${ }^{3}$ Idaho National Laboratory, Nuclear Materials Department, Idaho Falls, ID 83415, USA; ${ }^{4}$ Sandia National Laboratories, Materials Characterization and Performance, Albuquerque, NM 87185, USA; ${ }^{5}$ Sandia National Laboratories, Energetics Characterization, Albuquerque, NM 87185, USA; ${ }^{6}$ Aramco Research \& Development, Gharb Al Dhahran, Dhahran 34466, Saudi Arabia; ${ }^{7}$ Present address: Materials Department, University of California, Santa Barbara, CA 93106, USA

Correspondence: Michele L. Ostraat (michele.ostraat@aramcoservices.com)
}

Received: 18 September 2018 Accepted: 8 February 2019

Published online: 12 April 2019 
Real-time experimental observations of nanoscale solid-liquid interfacial processes are limited by the complexity of replicating a flowing corrosive environment within an instrument capable of characterizing corrosion initiation events that are rare, stochastic, and occur at the nanoscale. ${ }^{16,17}$ Previous studies targeting localized corrosion mechanisms have generally been conducted by examining the surfaces of steels ex-situ, using bulk-scale electrochemical testing in combination with surface characterization methods (e.g., scanning electron microscopy ${ }^{10}$ or scanning probe microscopy. ${ }^{18,19}$ ) Unfortunately, these techniques are too low in temporal and/or spatial resolution to conclusively identify the associated nanoscale mechanisms. Since its first demonstration in $2003,{ }^{20}$ liquid-cell scanning/transmission electron microscopy (LC-S/TEM) has undergone drastic improvements, now allowing for real-time structural and compositional characterization of materials within a flowing liquid environment. ${ }^{16,21-23}$ This technique has been used previously to study corrosion in model materials, such as nanoparticles and thin metal films. ${ }^{24,25}$ The use of in-situ LC-S/TEM to study more complex, less ideal "real world" alloys has only recently begun. ${ }^{26}$

Here, we present the application of in-situ LC-STEM to study localized corrosion in 1018 carbon steel during exposure to a flowing, aqueous solution. This study was designed to elucidate the mechanism by which localized corrosion of steel at cementite inclusions occurred by relating the known microstructure to localized electrochemical processes resolved by in-situ observation. Advanced structural and analytical electron microscopy techniques were combined to obtain time-resolved, site-specific nanoscale imaging of localized corrosion processes occurring simultaneously at the many different solid-liquid interfaces present in a "real world" sample of pipeline steel. In-depth structural and compositional analysis provided the deeper understanding necessary for studying the stochastic initiation events associated with localized corrosion.

A new experimental methodology and workflow (Fig. 1) was developed that paired in-situ, real-time data with extensive initial (preliminary) and final (postmortem) structural and compositional data sets in order to identify the critical nanoscale features in the steel microstructure that were most susceptible to localized corrosion. An electron-transparent sample was prepared from the bulk exterior of a 1018 steel industrial pipe using traditional focused ion beam (FIB) milling and lift-out procedures ${ }^{27}$ (see Methods). In preliminary examination (ex-situ), full characterization of the microstructure was performed using bright-field (BF) and high angle annular dark-field (HAADF) STEM to image the initial microstructure, including grain boundary locations. Grain orientation mapping with precession electron diffraction (PED) ${ }^{28}$ was used to identify the microstructure, including distinguishing cementite (orthorhombic crystal structure) from ferrite (bodycentered-cubic crystal structure) and quantifying relative misorientation angles. In addition, several localized initial thickness measurements were collected using electron energy loss spectroscopy $(E E L S)^{22}$ for later quantification of the total corrosioninduced thickness change in the Z-plane via comparison to postmortem data. Elemental composition examination was performed for select regions of interest around the known cementite grains using X-ray energy dispersive spectroscopy (XEDS, see SI Fig. S5). Collectively, these data were used to elucidate the complex microstructural relationships that governed where and how accelerated, localized corrosion initiated and progressed. This level of detail was necessary to simultaneously study the many possible initiation sites in the sample.

For the in-situ LC-STEM corrosion experiment, the sample was placed between two electron-transparent silicon nitride windows, forming a well-controlled liquid cell. The cell was pre-loaded with a droplet of deionized water to ensure complete wetting of the sample surface and was then placed into a liquid flow holder. The holder was then loaded into a transmission electron microscope, and microfluidic pumping $(2 \mu \mathrm{L} / \mathrm{min})$ of an aqueous electrolyte (pH 6.1) containing $\mathrm{CO}_{2}(a q ., 6 \mu \mathrm{M}), \mathrm{O}_{2}(a q ., 281 \mu \mathrm{M})$, and $\mathrm{Na}_{2} \mathrm{SO}_{4}$ (aq., $2.78 \mu \mathrm{M}$ ) was initiated. This solution composition was chosen to simulate an aqueous, baseline corrosive environment employing atmospheric levels of aqueous gases and a minimal amount of buffering salt to maintain $\mathrm{pH}$. Solution flow was maintained with no solution recirculation to ensure that reactants were not depleted. Exposure times given herein indicate the total time

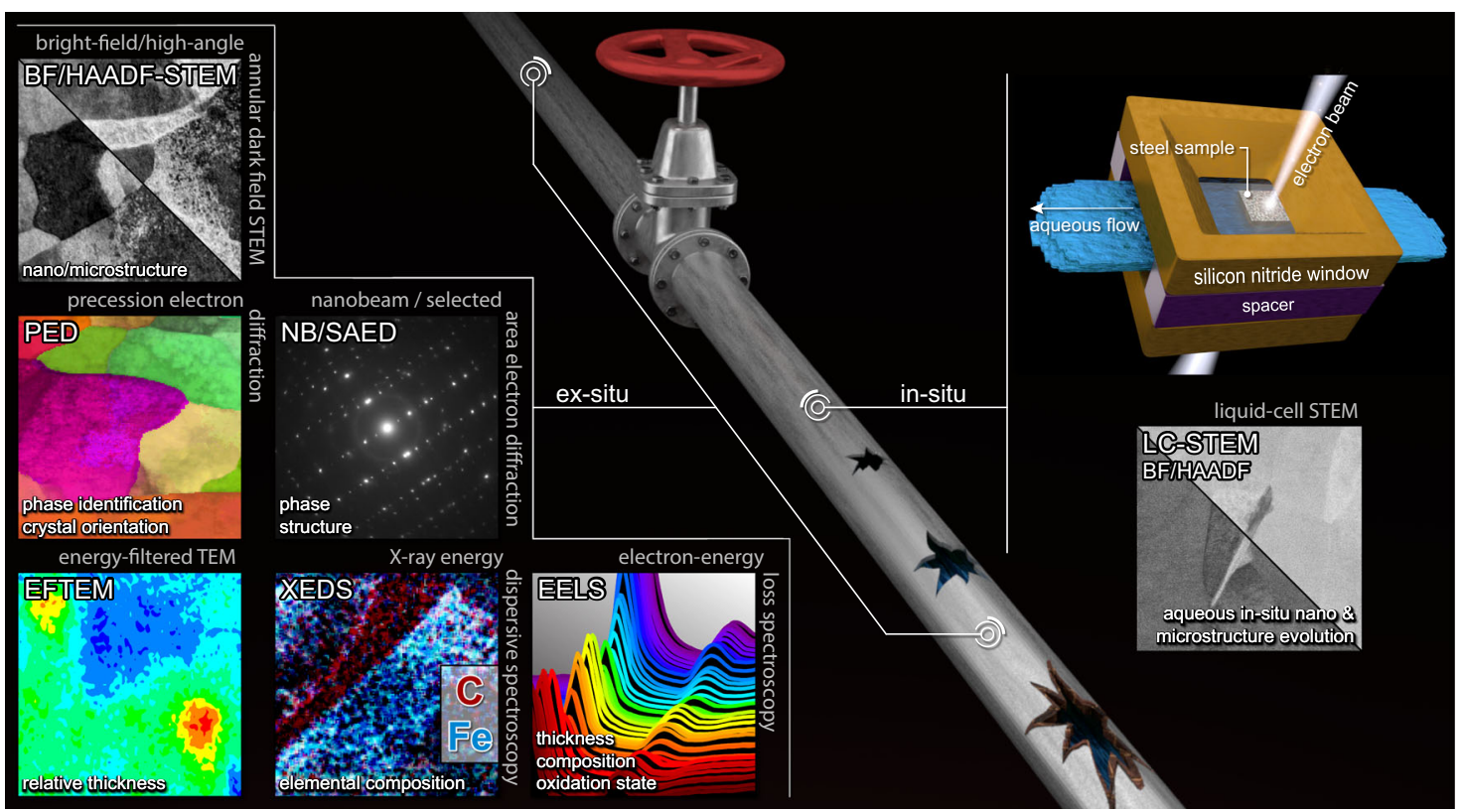

Fig. 1 Experimental workflow for examining aqueous corrosion initiation in 1018 steel in real time at the nanoscale. The initial sample was characterized using a suite of electron microscopy techniques, whose names, abbreviations, primary data types, and representative data sets are provided. The fully characterized sample was placed in a liquid cell for in-situ real-time observation under aqueous flow. The corroded sample was then characterized using the same suite of ex-situ electron microscopy techniques to aid in the interpretation of the in-situ observations 
the sample was exposed to aqueous electrolyte; fluid flow initiated after $10 \mathrm{~min}$ of static liquid exposure. STEM imaging was performed to record the corrosion processes in real time. Images were acquired sparingly over a $93 \mathrm{~min}$ period using low electron-fluence conditions, and the beam was blanked between exposures to minimize the production of-and interference byradiolysis products in the aqueous environment. ${ }^{29}$ The overall electron dose was kept well below the threshold at which other groups have observed interference by radiolysis products (see SI for more details). ${ }^{30}$ The corrosion experiment was concluded after 1025 min exposure to aqueous electrolyte. Extensive postmortem characterization was conducted on the corroded steel sample to ascertain (1) the final thickness using EELS and energy-filtered transmission electron microscopy (EFTEM), (2) the final microstructure using S/TEM and nanobeam electron diffraction (NBED), (3) the final composition using XEDS and EELS, and (4) the final oxidation state of scale products using EELS. The initial, transient, and final microstructures were compared using image averaging and image overlays to identify active localized corrosion sites and to determine the relative corrosion dissolution rates of the ferrite and cementite.

\section{RESULTS}

Localized initiation

One region near the center of the steel specimen displayed clear indications of localized accelerated corrosion. Crystallographic data (Fig. 2a) and BF-STEM micrographs of this region before (Fig. 2b) and during (Fig. 2c-f) exposure to aqueous electrolyte are provided. The first evidence of localized corrosion was visible after $40 \mathrm{~min}$ of exposure to liquid electrolyte (Fig. 2c), indicated by rapid changes in intensity in the in-situ STEM micrographs. Crossreferencing these brighter areas with the preliminary structural data (Fig. 2a, b) revealed that the initiation event occurred at the triple junction formed by an isolated cementite grain inclusion $\left(\mathrm{Fe}_{3} \mathrm{C}\right)$ and two abutting ferrite grains $(\mathrm{a})$, identified as $\mathrm{aA}$ and $\mathrm{aB}$ (Triple Junction 1, TJ1). Crystallographic analysis indicated zone axis orientations (direction out of plane) of [ $\overline{2} 0 \overline{5}]$ and [619] for grains $\mathrm{aA}$ and $\mathrm{aB}$, respectively, for a disorientation angle of 11 degrees across the ferrite grain boundary (see SI for full table of ferrite Euler angles). The cementite grain had a zone axis orientation of $[\overline{2} 9 \overline{5}]$ (orthorhombic space group Pbnm, where $\mathrm{a}<\mathrm{b}<\mathrm{c})$.

\section{Localized progression}

Following initiation at TJ1, localized corrosion progressed over the electrochemically active phase boundary formed by the interface between the cementite grain and its neighboring ferrite grains. Over the course of the 5 mins that elapsed between Fig. $2 c$ and $d$, evidence of continued localized corrosion was apparent in the BFSTEM micrographs (Fig. 2d), as the material corroded both at the triple junction formed by $\mathrm{Fe}_{3} \mathrm{C} / \mathrm{aB} / \mathrm{aC}$ (TJ2) and at the triple junction formed by $\mathrm{Fe}_{3} \mathrm{C} / \mathrm{aE} / \mathrm{aF}$ (TJ3). Grain aC was oriented along the [618] zone axis, with 5 degrees disorientation relative to $\mathrm{aB}$. Grains $\mathrm{aE}$ and $\mathrm{aF}$ had a similarly low relative disorientation angle

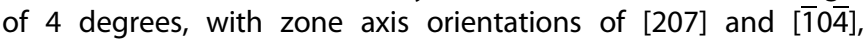
respectively. The accelerated mechanism laterally claimed up to $35 \mathrm{~nm}$ of ferrite (at TJ1) from grains $a A / a B$, after which the accelerated material loss arrested (Fig. 2e).

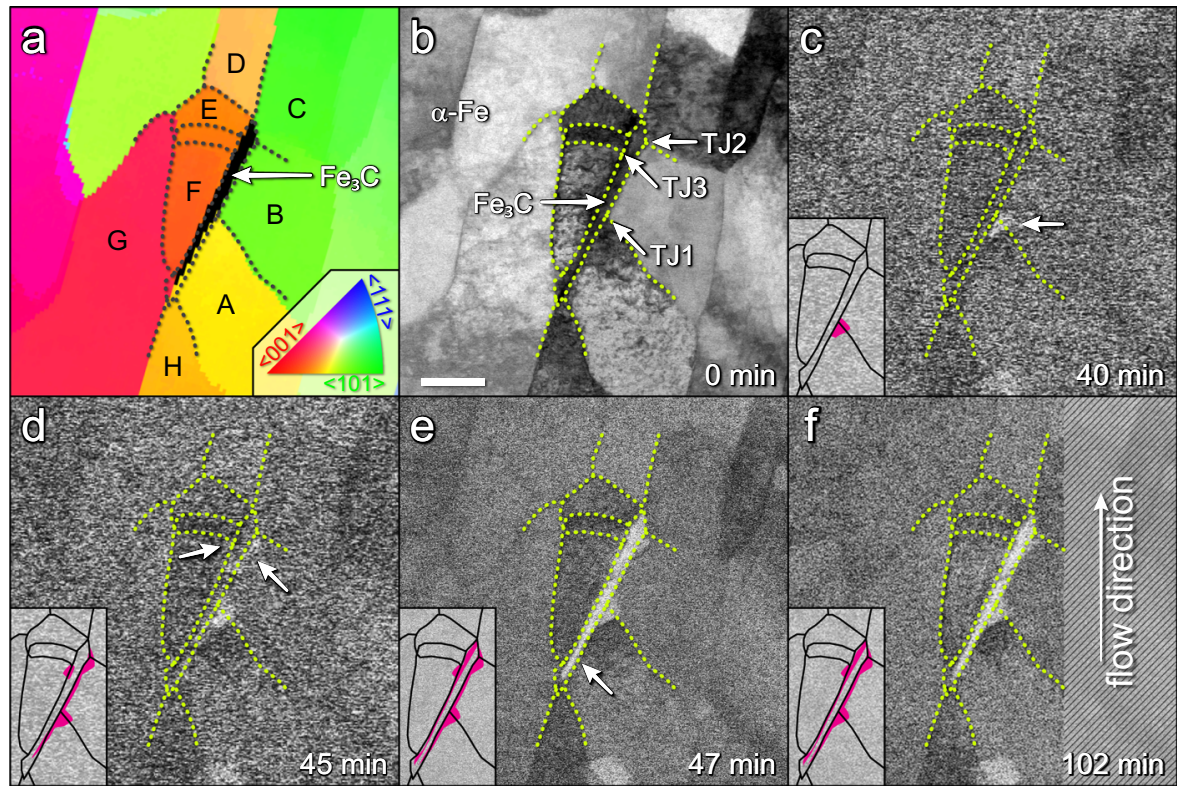

Fig. 2 Aqueous corrosion of 1018 steel visualized by in-situ STEM. Scale bar indicates $200 \mathrm{~nm}$. The PED crystallographic orientation map and bright-field STEM micrographs depict the region where localized corrosion was observed. Liquid exposure times are provided in each panel. Regions of increasing intensity resulted from increased transmission of the beam (c-f) and correspond to localized corrosion events. Grain boundaries are delineated in yellow to facilitate comparison between panels, and insets show a schematic of the grain boundaries with regions experiencing localized corrosion highlighted in pink. a PED map indicating the various crystallographic orientations in the bodycentered cubic ( $\alpha$-ferrite) reference plane. Black pixels denote non-BCC phases (i.e., cementite). b BF-STEM micrograph showing the microstructure of this region in vacuum prior to exposure to the aqueous electrolyte; the cementite grain inclusion was completely bordered by a ferrite matrix. TJ1, TJ2, and TJ3 are indicated with white arrows. c-f BF-STEM micrographs depicting this region during exposure to flowing aqueous solution. $c$ Localized corrosion at the inclusion was first observed at the triple junction (white arrow) between $\mathrm{Fe}_{3} \mathrm{C}$, $\alpha \mathrm{A}$, and $\alpha B$ (TJ1). Total electron flux $0.905 \mathrm{e}^{-} / \AA^{2 *}$ s. d At $45 \mathrm{~min}$, localized corrosion was observed to have claimed the interface between the Fe ${ }_{3} \mathrm{C}$ grain and grains $\alpha \mathrm{A} / \alpha \mathrm{B} / \alpha \mathrm{C}$, as well as the triple junctions (white arrows) formed by $\mathrm{Fe}_{3} \mathrm{C} / \alpha \mathrm{B} / \alpha \mathrm{C}$ (TJ2) and Fe $\mathrm{C}_{3} \mathrm{C} / \alpha \mathrm{E} / \alpha \mathrm{F}$ (TJ3). Total electron flux $0.915 \mathrm{e}^{-} / \AA^{2 *}$ s. e At $47 \mathrm{~min}$, the localized corrosion area stopped evolving. Total electron flux $1.23 \mathrm{e}^{-} / \AA^{2 *}$ s. f Final in-situ micrograph acquired after $102 \mathrm{~min}$ of solution exposure. Total electron flux $5.15 \mathrm{e}^{-} / \AA^{2 *} \mathrm{~s}$ 
Localized arrest

No further progression of localized corrosion was observed between 47 and 102 min, when in-situ observation concluded;

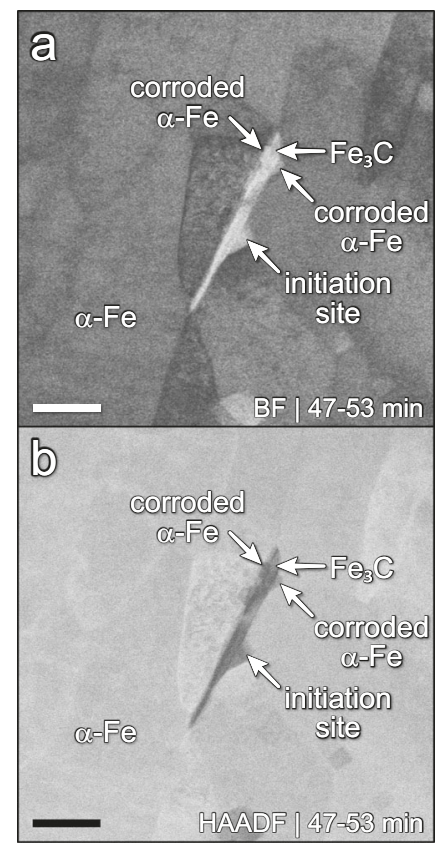

Fig. 3 Examination of 1018 steel region that experienced localized corrosion. Scale bar indicates $200 \mathrm{~nm}$. Aligned and averaged in-situ a BF-STEM and b HAADF-STEM images showing accelerated corrosion at the phase boundary between ferrite and cementite. Four images over a $6 \mathrm{~min}$ period were averaged the corroded area in Fig. 2e and $f$ was measured to be the same within the observation error of the experiment $\left( \pm 2 \mathrm{~nm}^{2}\right)$. To facilitate investigation of the microstructure after localized corrosion arrested, image averaging was used to increase the signal-to-noise ratio of the in-situ image data. While initial examination of single in-situ STEM micrographs (Fig. 2c-f) suggested that the $\mathrm{Fe}_{3} \mathrm{C}$ corroded away during this period, analysis of averaged BF (Fig. 3a) and HAADF STEM (Fig. 3b) micrographs from 47 to 53 min revealed that corrosion at the phase boundary only occurred in ferrite. Diffraction contrast changes in the cementite grain and removal of ferrite from the inclined phase boundary in relation to the observation direction account for the intensity variations co-localized with the cementite grain position. The arrest of localized corrosion at 47 min occurred either by the loss of physical contact between cementite and ferrite, or by passivation of the area by deposited corrosion product (vide infra).

\section{Final microstructure}

Postmortem TEM micrographs (Fig. 4a, b) revealed a crevice in the region of the solitary cementite inclusion. The crevice had grown outward along the grain boundary at the top of the inclusion (as shown) between grains $\mathrm{aC}$ and $\mathrm{aD}$ and at the bottom of the inclusion between grains $a \mathrm{G}$ and $\mathrm{aH}$ (Fig. 2a). The material occupying the gap within the crevice was characterized as corrosion product that overlapped the cementite inclusion in its original position. Rapid galvanic corrosion at this phase boundary likely induced a local spike in the concentration of solvated iron ions, leading to preferential deposition of corrosion product in the area. Product deposition increased with proximity to the cementite grain. Linear profiles extracted from the EFTEM intensity map (Fig. 4c) show that the corrosion product deposition on either side of the crevice was roughly evenly distributed, indicating that product deposition was independent of fluid flow. Contrast in the $\mathrm{BF}$ and HAADF postmortem S/TEM micrographs also revealed the
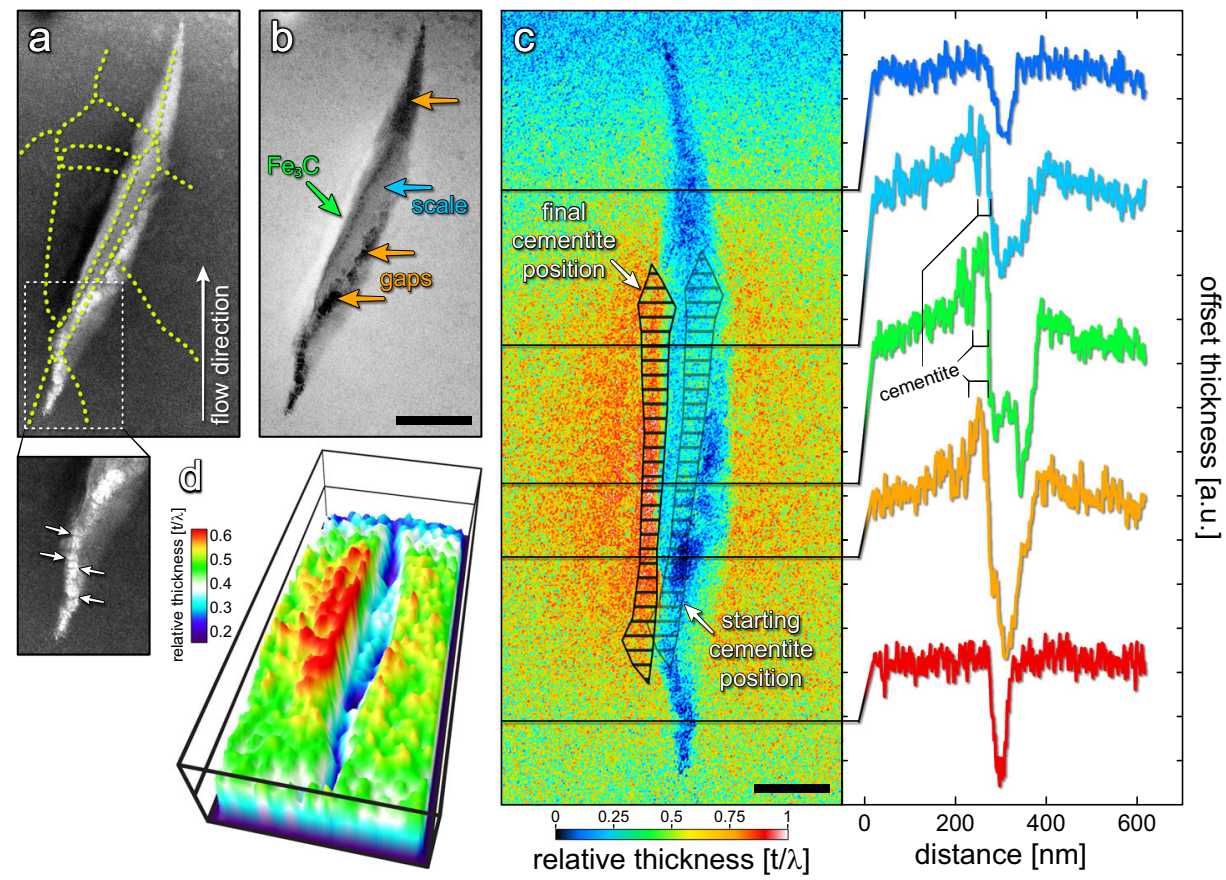

Fig. 4 Postmortem examination of 1018 steel region that experienced localized corrosion. Overlay of original grain boundaries on a postmortem a TEM image, with inset of crevice tip and arrows indicating the positions of bridging iron oxide structures observed in postmortem analysis, and $\mathbf{b}$ inverted TEM image of the crevice region showing corrosion product formation within and around the crevice. Scale bar indicates $200 \mathrm{~nm}$. c EFTEM relative thickness map of the crevice region depicting increased local corrosion product formation surrounding the crevice. Outlines of the initial (gray) and final (black) cementite inclusion location are provided. Scale bar indicates $100 \mathrm{~nm}$. d $3 D$ representation of matrix-smoothed EFTEM relative thickness map, highlighting the final location of the cementite grain 
presence of bridging structures - determined via elemental and crystallographic analysis to be amorphous iron oxide - connecting the two walls of the crevice (Fig. 4a, inset), which may have implications for corrosion product deposition/dissolution mechanisms. Further understanding of the corrosion product deposition mechanisms could be achieved by implementing new instrumentation and methods that are complementary to in-situ TEM and that enable tracking of the iron oxide evolution in-situ. ${ }^{22,31}$

Overlaying the initial grain boundary outline onto the postmortem image (Fig. $4 \mathrm{a}$, b) revealed that the cementite grain had moved relative to its initial position (confirmed via TEM and spot diffraction analysis), rotating from its initial 25 degrees to a final 20 degrees relative to the fluid flow direction (Fig. 4c, d). This movement indicated that corrosion at the phase boundary advanced sufficiently during the unobserved portion of the experiment to liberate the cementite grain from its original position in the ferrite matrix while leaving it confined within the crevice. A dip in intensity was visible to the left of the displaced cementite inclusion in both the HR-S/TEM micrographs (Fig. 4b) and the relative thickness map (Fig. $4 \mathrm{C}$ ). This intensity change is indicative of re-initiation of galvanic corrosion at the interface between the two phases, which occurred when the cementite was reconnected to the ferrite prior to the ferrite being fully converted to corrosion product.

\section{Uniform corrosion}

Running concurrent to the localized, accelerated corrosion discussed above, much slower, uniform corrosion was also observed over all the ferrite grains present in the sample. Fig. 5 shows overview S/TEM images of the entire steel coupon in vacuum before (Fig. 5a) and after (Fig. 5b) exposure to the liquid electrolyte. Initial EELS relative thickness measurements and EFTEM relative thickness maps were used to determine the average overall iron depletion to be between 70 and $85 \%$ over the course of the experiment (Fig. 5c). Electron diffraction and EELS analysis determined that all ferrite was converted to iron oxide (see $\mathrm{SI}$ ) by the end of the experiment. EELS relative thickness measurements in the pearlite grain present in the bottom left of the sample (Fig. 5b-inset) illustrate the preferential corrosion of ferrite and the relative resistance of cementite to corrosion. The starting thickness of all grains in this region was $85 \mathrm{~nm}$ : the corrosion-resistant cementite grains underwent no change in thickness, whereas the corroded ferrite regions returned a final thickness of $55 \mathrm{~nm}$ (indexed as $\mathrm{Fe}_{2} \mathrm{O}_{3}$ ).

\section{DISCUSSION}

Corrosion product formation and material loss during uniform corrosion are generally nonlinear in rate; significantly higher corrosion rates are often observed over the first several hours of uniform corrosion, followed by a steady-state rate once the iron surface has been converted to corrosion product. ${ }^{32}$ Qualitatively, we determined that the steel sample, which may have been exposed to the solution on both sides of the sample, was not completely converted to corrosion product within $102 \mathrm{~min}$ of exposure. This was evident in the in-situ image acquired at 102 min (Fig. 2f), where observable diffraction contrast within the ferrite grains (due to crystallographic orientation differences) indicated the presence of crystalline ferrite. However, these diffraction contrast differences were not present at the conclusion of aqueous exposure; postmortem EELS and XEDS revealed that the ferrite was fully converted to amorphous corrosion product following the $1025 \mathrm{~min}$ of exposure (Figs $4 \mathrm{a}$, b and $5 \mathrm{~b}$ ). At this point, the ferrite regions of the sample were observed to have lost ca. $30 \mathrm{~nm}$ of thickness, retaining $55 \mathrm{~nm}$ in the form of iron oxide. Given the starting thickness of $85 \mathrm{~nm}$ and the quantitative ferrite dissolution timeline, the corrosion material loss rate due only to

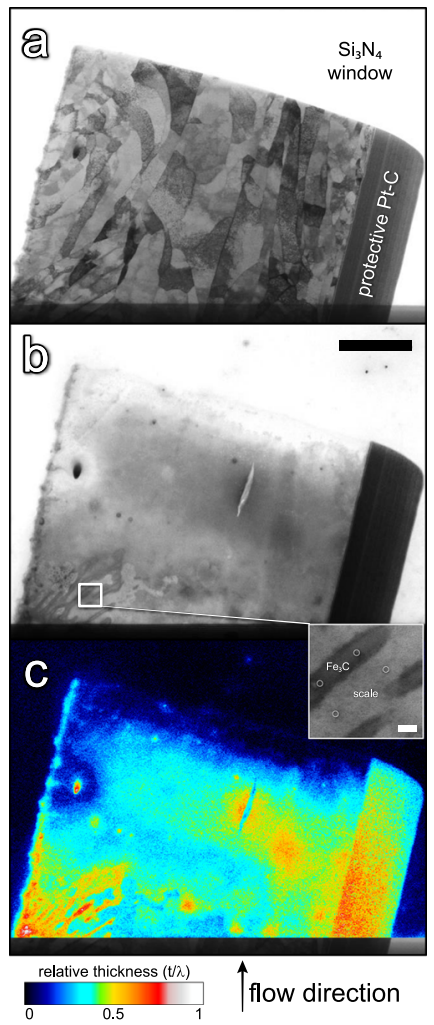

Fig. 5 Analysis of uniform aqueous corrosion of 1018 steel. a BFSTEM micrograph showing steel sample on a single liquid-cell window prior to exposure to aqueous media. $\mathbf{b}$ TEM micrograph of corroded sample on a single liquid-cell window. Scale bar indicates $2 \mu \mathrm{m}$. Inset, circles identify locations of corresponding EELS log-ratio thickness measurements for the layered ferrite-cementite grains, as discussed in the text. Inset scale bar indicates $100 \mathrm{~nm}$. c EFTEM relative thickness map of corroded sample with $\mathrm{Si}_{3} \mathrm{~N}_{4}$ window thickness subtracted

uniform corrosion was calculated to be between 0.015 and $0.16 \mathrm{~mm}$ per year, with a depth of penetration between 0.044 and $0.44 \mathrm{~mm}$ per year. These values are expected to be an upper bound, as they were calculated assuming possible contact of both sample surfaces with the solution.

Previous experimental workflows have struggled to separate the contributions of uniform and localized processes to the overall corrosion rate; LC-TEM provides a finite sample volume with transmission imaging that is able to indicate changes in thickness and material composition. While discrepancies may arise due to differences in the steel surface roughness and density of surface phase boundaries between bulk and nanoscale samples, the ability to decouple the contribution of localized and uniform processes could be a major boon to the development of more robust corrosion prediction models capable of accurately modeling the individual mechanisms responsible for material degradation.

It has been previously demonstrated that the final morphology of low-carbon steel after attack by aqueous solution exhibited surface structures described as "soft line form" features: widely spaced, small-aspect-ratio ridges. ${ }^{33}$ The results of this work indicate that these soft line microstructures may result from two concurrent processes: (1) release of cementite grains following complete dissolution of the phase boundary between cementite and ferrite, leaving exposed troughs, and (2) increased corrosion product deposition in regions where this type of localized corrosion occurs. The combination of these two events would generate lined crevices with softened features in the regions where cementite grains were released. This realization was 


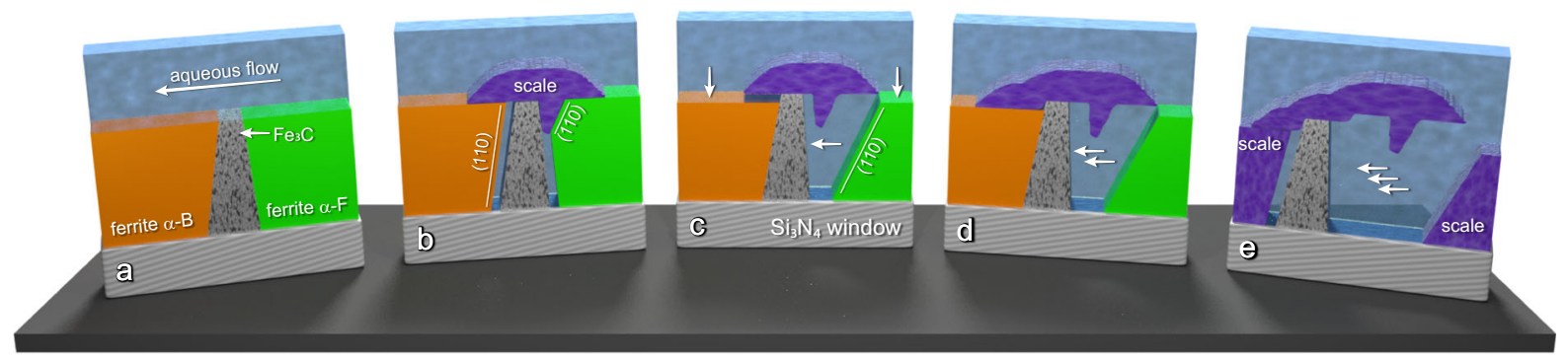

Fig. 6 The post-corrosion landscape and characterization data suggest a reinitiating galvanic mechanism represented by this schematic illustration. a Cross-section of the localized corrosion region with the cementite grain surrounded by ferrite and exposed to an aqueous solution. b Ferrite at the phase boundary corroded, and the resulting local spike in solvated iron species caused corrosion product to form. $\mathbf{c}$ Corrosion of ferrite progressed significantly enough to expose the cementite grain to fluid flow and to allow fluid access to the cavity, which pushed the cementite grain into grain $\alpha B$. $\mathbf{d}$ The reconnected cementite-ferrite interface reinitiated galvanic corrosion. e Galvanic corrosion terminated due to loss of cementite mobility and/or severance of electrochemical activity, as ferrite was fully converted to corrosion product by the end of the experiment. Incident beam direction from bottom as pictured

achieved due to real-time, in-situ transmission-mode imaging of the sample at the nanoscale, which captured finite surface-level processes that were previously undetected.

Characterization data and key observations suggest that a reinitiating mechanism may be involved in the deleterious interaction between the cementite inclusion and the neighboring, receded ferrite grains. Detailed image analysis contrasting the last of the in-situ images with the postmortem images of the localized corrosion region identified relocation of the cementite grain (bright region indicated in Fig. 4b) as well as an intensity decrease along the left side of the final position of the cementite grain (visible as a slightly darker region to the left of the bright cementite in Fig. 4b and in the line profiles in Fig. 4c). We propose a schematic of this re-initiation process in Fig. 6. Localized corrosion occurred first at the TJs and phase boundaries (vide supra), leading to faceted pitting, and the spike in local iron ion concentration led to preferential accumulation of corrosion product in the area (Fig. $6 \mathrm{~b}$ ). During the unmonitored portion of aqueous exposure, the corrosion-resistant cementite grain was unaffected, but the ferrite grains etched in the $Z$ direction (Fig. $6 \mathrm{c}$ ), leading to gaps in the corrosion product layer that were highlighted by the TEM micrographs and relative thickness maps (Fig. 4c, d). The formation of these gaps both allowed the fluid to regain access to the cavity and left the cementite inclusion jutting out from the surface. The final position of the cementite grain suggests that the fluid flow pushed the cementite grain, which was oriented at $25^{\circ}$ to the flow direction, into $\mathrm{aE} / \mathrm{aF}$, reinitiating accelerated galvanic corrosion (Fig. $6 \mathrm{~d}$ ) and leading to the dip in intensity seen in the relative thickness map to the left of the final inclusion position (Fig. 4c) as the reconnected cementite and ferrite grains once again experienced localized corrosion at their phase boundary. This process would have continued until either the interface was no longer electrochemically active (ferrite conversion to corrosion product) and/or the cementite grain lost mobility (Fig. 6e), ultimately leading to the same result: a rotated, confined cementite inclusion with a distinct intensity dip to its left in the thickness map.

The labile nature of the cementite grain-following the localized-corrosion-mediated dissolution of its surrounding ferrite matrix at the phase boundaries-has far-reaching implications. On the bulk scale, these types of inclusions and phase boundaries are highly prevalent in the steel microstructure. Cavities formed around them via the mechanism observed here would not only allow infiltration of the corrosive medium into the inner structure of the steel, but the dislodged cementite grains could re-initiate galvanic corrosion in any ferrite regions of the steel surface to which they were displaced. Additionally, turbulent fluid flow combined with a cementite grain trapped within a ferrite cavity could lead to a larger opening within the steel subsurface by the same deleterious mechanism.

The localized corrosion at the TJs along the phase boundary of the solitary cementite inclusion (e.g., Fig. 2c, arrow) showed faceted pitting of the corroded ferrite. Identification of these ferrite pit facets was determined by comparing the faceted edge of the etched region (Fig. 2f) with possible faceted pit geometries, their 3D intersection with the ferrite surface, and the ways these would appear in a 2D S/TEM micrograph projection. Each of the pits along the phase boundary of the solitary inclusion was found to have $\{110\}$ faceting. In comparison, no pitting was observed at the cementite inclusions in the pearlite grain, which did not exhibit any obvious TJs. This suggests that structural defects, such as TJs, are important factors in the initiation of localized corrosion. Structural defects may indicate increased matrix strain and/or increased accommodation of dopants that can increase the activity at these sites. TJ1 was likely not the most strained or defected site along the phase boundary. If structural defects were the only contributor to the onset of localized corrosion, then the junction formed by the intersection of four grains $\left(\mathrm{Fe}_{3} \mathrm{C}, \mathrm{aC} / \mathrm{aD} / \mathrm{aE}\right)$ might be expected to corrode first. Instead, the initiation event occurred at TJ1, located at approximately mid-length of the $\mathrm{Fe}_{3} \mathrm{C}$ inclusion and having the highest contact area to the cathodic inclusion of any grain-boundary-based defect. Furthermore, TJ2 and TJ3 were located about the same distance along the length of the cathodic inclusion and pit at the same time, despite the TJ2 ferrite surfaces being more favorably oriented for pitting over TJ3. ${ }^{34}$ This may indicate that some interplay between galvanic and strain-based mechanisms is responsible for the pitting behavior observed here.

We observed the advent of localized steel corrosion in-situ and identified the nanoscale features implicit in the initiation and progression of an accelerated corrosion mechanism. Localized corrosion initiated at a site of interfacial heterogeneity: a triple junction formed by the intersection of two ferrite grains and an isolated cementite inclusion. The initiation event involved two ferrite grains with a relatively long surface contact length with the cementite inclusion and occurred at the most structurally defected site along the phase boundary involved in these three grains. Following initiation, accelerated corrosion progressed along the electrochemically active interface formed by the ferrite-cementite phase boundary, claiming adjoining portions of abutting ferrite grains, leaving the cementite composition unaffected, and trapping the dislodged cementite grain within the resulting cavity. On the bulk scale, such a cavity could allow infiltration of the corrosive medium into the inner structure of the steel and could feasibly serve as the initiation point of a crack. The isolated cementite grain was not the only cementite grain present in the sample; however, it was the only cementite grain that exhibited 
clear signs of localized, accelerated corrosion, and it was the only cementite inclusion that displayed clear triple junctions along the phase boundary. Comparison of the relative activity of the pitted locations along the solitary inclusion suggests that a combined galvanic and strain-based mechanism may be responsible for the localized corrosion observed in this work.

The experimental workflow and array of electron microscopy techniques established herein allowed for the observation of stochastic, nanoscale events using non-ideal, field-relevant materials and conditions. TEM is particularly suited to this type of investigation, as it provides elemental composition, crystallographic orientation, and nanoscale observation of dynamic events and transient states that could pass unobserved in techniques with lower temporal and spatial resolution. Additionally, other techniques that are primarily sensitive to topology (e.g., scanning electron microscopy or atomic force microscopy) would be limited in this endeavor, as corrosion product deposition concurrent with accelerated corrosion would generate a surface in which any percolation pathways would be invisible to such surface-sensitive techniques. The insight necessary to interpret the in-situ LC-STEM data provided by initial and postmortem analysis emphasizes the importance of full-spectrum characterization to support a mechanistic interpretation. Future investigations will use an approach that specifically targets the active electrochemical interfaces in steel whose involvement in localized corrosion was implicated in this work. New hardware and techniques are being implemented to obtain real-time compositional information from materials within the LC-STEM concurrent with in-situ observation. $^{23,31}$

Nanoscale corrosion pathways, like the one identified in this work, could have a profound impact on the degradation behavior of bulk materials. This work suggests that the types of electrochemically active interfaces that typify and pervade the steel microstructure may participate in accelerated corrosion mechanisms that, extrapolated to the bulk-scale, would create percolation networks that expose the interior body of the steel to corrosive conditions much sooner than predicted by uniform corrosion models. These types of scientific insights will prove vital for the development of more robust corrosion prediction models as well as the development of next-generation corrosion prevention and mitigation strategies.

\section{METHODS}

Low-carbon steel sample

A small coupon (ca. $12.7 \times 12.7 \times 3.175 \mathrm{~mm}$ ) of cold finish mild steel, SAE/ AISI 1018, produced by the manufacturer S. Izaguirre, S.A, was used for characterization and corrosion testing. The bulk chemical composition provided by the manufacturer is (wt\%): $0.16 \mathrm{C}, 0.71 \mathrm{Mn}, 0.169 \mathrm{Si}, 0.016 \mathrm{P}$, $0.021 \mathrm{~S}, 0.116 \mathrm{Cr}, 0.107 \mathrm{Ni}, 0.017 \mathrm{Mo}, 0.345 \mathrm{Cu}, 0.017 \mathrm{Sn}, 0.008 \mathrm{Al}, 0.002 \mathrm{~V}$, $0.0088 \mathrm{~N}, 0.001 \mathrm{Ti}, 0.002 \mathrm{~Pb}$, and balanced by Fe. The coupon was coated with a thin layer of grease (Starrett M1 Oil) to preserve the surface state during storage.

\section{Focused ion-beam TEM sample preparation}

The steel coupon was affixed to a scanning electron microscope (SEM) stub after removal of the grease layer using isopropyl alcohol. An FEI Nova NanoLab $^{\text {TM }} 600$ DualBeam $^{\text {TM }}$ focused ion-beam(FIB)/SEM was used to deposit a ca. $100 \mathrm{~nm}$ thick protective layer of $\mathrm{Pt} / \mathrm{C}$ (ca. 83/17 at $\%{ }^{35}$ ) with a $15 \mathrm{kV}$ electron beam over a ca. $2 \times 20 \mu \mathrm{m}$ area of the coupon surface followed by a ca. $1 \mu \mathrm{m}$ thick protective layer of $\mathrm{Pt} / \mathrm{C}$ with a $30 \mathrm{kV}$ Ga ion beam prior to $30 \mathrm{kV}$ Ga ion milling of the foil. An Omniprobe micromanipulator was then used for in-situ lift-out of the lamella to an Omniprobe Cu transmission electron microscope (TEM) half-grid. The sample was thinned to electron transparency and then received a final $10 \mathrm{kV}$ Ga ion thinning step to reduce the Ga-ion-damaged surface layer. The sample was characterized prior to corrosion testing in this configuration.
For in-situ TEM corrosion testing, the steel lamella was removed from the Omniprobe half-grid, again using standard FIB lift-out techniques with $30 \mathrm{kV} \mathrm{Ga}$ ion beam cuts, and transferred to the edge of a $50-\mathrm{nm}$ thick silicon nitride $\left(\mathrm{Si}_{3} \mathrm{~N}_{4}\right)$ window of a TEM liquid-cell chip (Hummingbird Scientific), such that the lamella rested partially on the thick Si at the window edge and partially overhung the window.

\section{Characterization of initial structure and composition}

The initial 1018 steel lamella microstructure was characterized using precession electron diffraction (PED) phase and orientation mapping. The PED data was acquired using a NanoMEGAS DigiSTAR ${ }^{\mathrm{TM}}$ precession unit installed on a $200 \mathrm{kV}$ JEOL 2100 TEM with a $7 \mathrm{~nm}$ probe size over a $3.3 \times$ $3.8 \mu \mathrm{m}$ area with $10 \mathrm{~nm}$ steps and indexed using ASTAR $^{\text {TM }}$ (NanoMEGAS) software. Sample composition was characterized using X-ray energy dispersive spectroscopy (XEDS). The XEDS maps were acquired on a $200 \mathrm{kV}$ FEI Titan ChemiSTEM equipped with 4 windowless silicon drift detectors. The XEDS maps were evaluated using multivariate statistical analysis with Automated eXpert Spectral Image Analysis (AXSIA) software. ${ }^{36}$ Relative sample thickness was measured in a grid of discrete points using the electron energy loss spectroscopy log-ratio technique ${ }^{23}$ on a $300 \mathrm{kV}$ Tecnai F30 G2 TEM, equipped with a Tridiem 863 Gatan Image Filter.

\section{Corrosion solution}

The liquid for the in-situ TEM corrosion experiment was an aqueous solution ( $\mathrm{pH}$ 6.1) containing atmospheric levels of dissolved gasses $(6 \mu \mathrm{M}$ $\mathrm{CO}_{2}, 287.5 \mu \mathrm{M} \mathrm{O}_{2}$ ) and sodium sulfate (Sigma Aldrich, $2.78 \mu \mathrm{M}$ ), prepared using ultrapure $\left(18.2 \mathrm{M} \Omega^{*} \mathrm{~cm}\right)$ water. Using a Hamilton syringe equipped with a stop-cock, $5 \mathrm{~mL}$ of the test solution was withdrawn and loaded onto the syringe pump for microfluidic pumping (at $2 \mu \mathrm{L} / \mathrm{min}$ ) throughout the experiment. The $\mathrm{CO}_{2}$ concentration was determined via titration with $\mathrm{NaOH}(0.1 \mathrm{mM})$ using phenolphthalein solution as an indicator. The $\mathrm{O}_{2}$ concentration was determined using a dissolved oxygen meter (VWR). The equilibrated water was prepared by allowing ultrapure water $\left(18.2 \mathrm{M} \Omega^{*} \mathrm{~cm}\right)$ to equilibrate under atmospheric conditions overnight and then adding the appropriate amount of sodium sulfate stock solution to reach $2.78 \mu \mathrm{M}$. Sodium hydroxide solution (Sigma Aldrich, $0.1 \mathrm{mM}$ ) was prepared from ultrapure $\left(18.2 \mathrm{M} \Omega^{*} \mathrm{~cm}\right)$ water.

\section{In-situ liquid-cell TEM experiment}

The liquid flow TEM holder (Hummingbird Scientific) assembly is composed of two liquid-cell chips, including a base and lid, that, when compressed and sealed within the holder, create an environmental cell with continuous liquid flow through a channel maintained with thin spacers. Each chip has a thin $\mathrm{Si}_{3} \mathrm{~N}_{4}$ window in the flow channel that permits electron transmission with minimal loss of spatial resolution while maintaining separation of the liquid from the vacuum of the TEM. A $250 \mathrm{~nm}$ SU-8 photoresist spacer, patterned onto the lid chip (as purchased), was used to provide the minimal fluid thickness within the cell before affixing the steel sample over the window. The base chip was plasma cleaned for $3 \mathrm{~min}$ just prior to in-situ TEM corrosion testing to produce a hydrophilic surface and to promote wetting and flow through the thin channel. However, the lid chip with the steel lamella was not plasma cleaned to avoid oxidation of the sample. The base and lid chips were loaded into the holder such that the electron beam would pass through the first window and sample before encountering the liquid to enable the highest STEM imaging resolution. The base chip was pre-loaded with a $6 \mu \mathrm{L}$ droplet of equilibrated deionized water to ensure complete wetting of the sample surface. Ten minutes elapsed between liquid loading and the acquisition of the first STEM image under solution flow.

The sample was imaged in STEM mode at $300 \mathrm{kV}$ using a beam current of $13 \mathrm{pA}$, and images were acquired with a $2 \mu \mathrm{s}$ dwell time. The sample was imaged over a $93 \mathrm{~min}$ period with images acquired about every minute at various magnifications of $2048 \times 2048$ pixels: 25 images at $4.06 \mathrm{~nm} / \mathrm{pixel}, 8$ images at $2.03 \mathrm{~nm} /$ pixel, 9 images at $1.44 \mathrm{~nm} /$ pixel, and 25 images at $1.02 \mathrm{~nm} /$ pixel were acquired with $0.1,0.4,0.8$, and $1.6 \mathrm{e}^{-} / \AA^{2}$ fluence at each image magnification, respectively. As the radiation dose to the specimen is dependent on the area scanned by the STEM probe, the magnification was kept low for most of the imaging, minimizing accumulated electron fluence in the region of the solitary cementite inclusion to $51.5 \mathrm{e}^{-} / \AA^{2}$. This was done to prevent the generation of radiation species that could alter the sample during observation. It should be noted that at the lowest magnification, the whole of the sample contained within the $\mathrm{Si}_{3} \mathrm{~N}_{4}$ window was imaged; therefore, reactions 
between the radiation species in the corrosion solution and on the sample surface would be evenly distributed over the entire sample. No preferential dosing would be expected, except at higher magnification, where the flyback in the scanning probe rests on the left side of the scanned image region.

The experiment continued unobserved overnight with solution flow maintained at $2 \mu \mathrm{L} / \mathrm{min}$. The solution was kept sealed, and a continuous flow of fresh solution was used throughout the experiment to ensure that reactant depletion would not affect the solution composition. The experiment was halted after $1025 \mathrm{~min}$ of total exposure to liquid. The sample was immediately removed from the liquid-cell holder, underwent a cursory removal of liquid via wicking with filter paper, and then was left to dry within a nitrogen dry box, where it was stored between postmortem analyses.

\section{Characterization of final structure and composition}

An initial survey of the corroded steel sample was performed on the Tecnai F30 TEM operated at $300 \mathrm{kV}$ to image the microstructure and measure the sample thickness. The corroded sample was affixed to the liquid-cell chip, imaged using BF/DF STEM, and the relative sample thickness expressed in units of mean free path were measured in a grid of discrete points based on the Fourier log-ratio EELS technique. More extensive sample thickness mapping was performed on the corroded sample using the same Fourier log-ratio technique with energy-filtered TEM using a Tridiem 863 Gatan Image Filter. Relative thickness maps (units of thickness divided by the material-dependent $300 \mathrm{kV}$ electron inelastic mean free path length) were acquired and calculated using DigitalMicrograph (Gatan) and further visualized with MATLAB.

Analytical transmission electron microscopy and core-loss energy loss spectroscopy was performed on a $200 \mathrm{kV} \mathrm{FEI} \mathrm{Titan} \mathrm{ChemiSTEM} \mathrm{equipped}$ with a Quantum Gatan Image Filter. High-resolution EELS chemical imaging was performed in STEM mode at $200 \mathrm{kV}$ with an emission current of $185 \mathrm{uA}, 3900 \mathrm{~V}$ extraction, $32 \mathrm{~mm}$ camera length, and $50.5 \mathrm{mrad}$ collection angle based on a 5-mm filter opening aperture. Energy windows extending from the oxygen- $K$ to iron- $L$ edge were collected using a dispersion of $0.25 \mathrm{eV} /$ pixel that was bias, gain, and drift corrected. A total of 70 individual spectra were collected along a line profile that were each individually collected for $10 \mathrm{~s}$. Using the $L_{3,2}$ white-line-ratio technique over the iron-L edge profiles, subtracted valence profiles were matched to experimental and published data for a variety of iron oxide scale products, including: $\mathrm{a}-\mathrm{Fe}_{2} \mathrm{O}_{3}, \mathrm{\gamma}-\mathrm{Fe}_{2} \mathrm{O}_{3}, \mathrm{a}-\mathrm{FeO}(\mathrm{OH}), \mathrm{Fe}_{3} \mathrm{O}_{4}, \mathrm{FeCO}_{3}$, and $\mathrm{FeO}$. Non-linear least squares peak fitting in addition to dual window and a 2:1 stepped background subtraction with lifetime broadening of the $L_{3,2}$ peaks was utilized to extract quantitative integrals associated with each peak and to calculate the profiles. Comparing experimental and published data fell within two standard deviations, owing to the analytical certainty associated with this core-loss EELS profiling technique.

\section{DATA AVAILABILITY}

The data that support the findings of this study are available from the corresponding author upon request.

\section{ACKNOWLEDGEMENTS}

This work was performed, in part, at the Center for Integrated Nanotechnologies, an Office of Science User Facility operated for the U.S. Department of Energy (DOE) Office of Science by Los Alamos National Laboratory (Contract DE-AC52-06NA25396) and Sandia National Laboratories (Contract DE-NA-0003525).

\section{AUTHOR CONTRIBUTIONS}

K.L.J., S.C.H., M.L.O., T.J.K. and C.C. designed the experimental methods. W.M.M. prepared the sample. C.C., J.A.A. P.G.K., D.C.B., K.H. and K.L.J. acquired experimental data. Data was interpreted by C.C., S.C.H., R.O.G., J.A.A., I.M.T., K.L.J. and M.L.O. The manuscript was prepared by S.C.H., C.C., R.O.G., T.S.P., K.L.J. and M.L.O.

\section{ADDITIONAL INFORMATION}

Supplementary Information accompanies the paper on the npj Materials Degradation website (https://doi.org/10.1038/s41529-019-0078-1).

Competing interests: The authors declare no competing interests.
Publisher's note: Springer Nature remains neutral with regard to jurisdictional claims in published maps and institutional affiliations.

\section{REFERENCES}

1. Hou, B. et al. The cost of corrosion in China. npj Materials Degradation 1, 4 (2017).

2. Kermani, M. \& Morshed, A. Carbon dioxide corrosion in oil and gas production-A compendium. Corrosion 59, 659-683 (2003).

3. De Waard, C. \& Milliams, D. Carbonic acid corrosion of steel. Corrosion 31, 177-181 (1975)

4. Nešić, S. Key issues related to modelling of internal corrosion of oil and gas pipelines-A review. Corros. Sci. 49, 4308-4338 (2007).

5. Significant Pipeline Incidents By Cause: Significant Incident Cause Breakdown: 20 Year Average (1997-2016). In Pipeline Incident 20 Year Trends. Updated: Wednesday, December 6, 2017 edn. (Pipeline and Hazardous Material Safety Administration, 2017)

6. Bhandari, J., Khan, F., Abbassi, R., Garaniya, V. \& Ojeda, R. Modelling of pitting corrosion in marine and offshore steel structures-A technical review. J. Loss. Prev. Proces.s Ind. 37, 39-62 (2015).

7. Revie R. W., Uhlig H. H. Uhlig's Corrosion Handbook. (John Wiley \& Sons, Hoboken, 2011).

8. Han, J., Yang, Y., Brown, B. \& Nesic, S. Electrochemical investigation of localized $\mathrm{CO}_{2}$ corrosion on mild steel. Corrosion 2007 Conference and Expo. Paper No. 07323 (2007).

9. Eklund, G. S. Initiation of pitting at sulfide inclusions in stainless steel. J. Electrochem. Soc. 121, 467-473 (1974).

10. Sun, J., Zhang, G., Liu, W. \& Lu, M. The formation mechanism of corrosion scale and electrochemical characteristic of low alloy steel in carbon dioxide-saturated solution. Corrosion Science 57, 131-138 (2012).

11. Wranglen, G. Pitting and sulphide inclusions in steel. Corrosion Science 14, 331-349 (1974).

12. Lillard S. Relationships Between Pitting Corrosion and Crystallographic Orientation, An Historical Perspective. Corrosion Science A Retrospective and Current Status in Honor of Robert P Frankenthal, 334-343 (2002).

13. Avci, R. et al. Mechanism of MnS-mediated pit initiation and propagation in carbon steel in an anaerobic sulfidogenic media. Corros. Sci. 76, 267-274 (2013).

14. Bertali, G., Scenini, F. \& Burke, M. The effect of residual stress on the preferential intergranular oxidation of alloy 600. Corros. Sci. 111, 494-507 (2016).

15. Staicopolus, D. The role of cementite in the acidic corrosion of steel. J. Electrochem. Soc. 110, 1121-1124 (1963).

16. Evans, J. E., Jungjohann, K. L., Browning, N. D. \& Arslan, I. Controlled growth of nanoparticles from solution with in situ liquid transmission electron microscopy. Nano. Lett. 11, 2809-2813 (2011).

17. Carter C., Williams D. Transmission Electron Microscopy - Diffraction, Imaging, and Spectrometry (Springer International Publishing Switzerland, Berlin, 2016).

18. Ulyanov, P. et al. Microscopy of carbon steels: Combined AFM and EBSD study. Appl. Surf. Sci. 267, 216-218 (2013).

19. Dwivedi, D., Lepková, K. \& Becker, T. Carbon steel corrosion: a review of key surface properties and characterization methods. RSC Advances 7, 4580-4610 (2017).

20. Williamson, M., Tromp, R., Vereecken, P., Hull, R. \& Ross, F. Dynamic microscopy of nanoscale cluster growth at the solid-liquid interface. Nat. Mater. 2, 532-536 (2003).

21. de Jonge, N., Poirier-Demers, N., Demers, H., Peckys, D. B. \& Drouin, D. Nanometer-resolution electron microscopy through micrometers-thick water layers. Ultramicroscopy 110, 1114-1119 (2010).

22. Jungjohann, K. L., Evans, J. E., Aguiar, J. A., Arslan, I. \& Browning, N. D. Atomicscale imaging and spectroscopy for in situ liquid scanning transmission electron microscopy. Microsc. Microanal. 18, 621-627 (2012).

23. Cho, $\mathrm{H}$. et al. The use of graphene and its derivatives for liquid-phase transmission electron microscopy of radiation-sensitive specimens. Nano. Lett. 17, 414-420 (2016).

24. Gross, D., Kacher, J., Key, J., Hattar, K. \& Robertson, I. M. In situ TEM observations of corrosion in nanocrystalline Fe thin films. Processing, Properties, and Design of Advanced Ceramics and Composites II 261, 329 (2017).

25. Chee, S. W. et al. Studying localized corrosion using liquid cell transmission electron microscopy. Chem.Communi. 51, 168-171 (2015).

26. Schilling, S., Janssen, A., Zhong, X., Zaluzec, N. \& Burke, M. Liquid In Situ Analytical Electron Microscopy: Examining SCC Precursor Events for Type 304 Stainless Steel in H 2 O. Microsc. Microanal. 21, 1291-1292 (2015).

27. Mayer, J., Giannuzzi, L. A., Kamino, T. \& Michael, J. TEM sample preparation and FIB-induced damage. MRS. Bull. 32, 400-407 (2007).

28. Rauch, E. F. et al. Automated nanocrystal orientation and phase mapping in the transmission electron microscope on the basis of precession electron diffraction. Zeitschrift für Kristallographie 225, 103-109 (2010). 
29. Schneider, N. M. et al. Electron-water interactions and implications for liquid cell electron microscopy. J. Phys. Chem. C 118, 22373-22382 (2014).

30. Woehl, T. \& Abellan, P. Defining the radiation chemistry during liquid cell electron microscopy to enable visualization of nanomaterial growth and degradation dynamics. J. Microsc. 265, 135-147 (2017).

31. Hart, J. L. et al. Direct detection electron energy-loss spectroscopy: a method to push the limits of resolution and sensitivity. Sci. Rep. 7, 8243 (2017).

32. Zhang, Y., Pang, X., Qu, S., Li, X. \& Gao, K. Discussion of the CO2 corrosion mechanism between low partial pressure and supercritical condition. Corros. Sci. 59, 186-197 (2012)

33. Clover, D., Kinsella, B., Pejcic, B. \& De Marco, R. The influence of microstructure on the corrosion rate of various carbon steels. J. Appl. Electrochem. 35, 139-149 (2005).

34. Kruger, J. Influence of crystallographic orientation on the pitting of iron in distilled water. J. Electrochem. Soc. 106, 736-736 (1959).

35. Botman, A., Mulders, J. \& Hagen, C. Creating pure nanostructures from electronbeam-induced deposition using purification techniques: a technology perspective. Nanotechnology. 20, 372001 (2009).
36. Kotula, P. G. \& Keenan, M. R. Application of multivariate statistical analysis to STEM X-ray spectral images: Interfacial analysis in microelectronics. Microsc. Microanal. 12, 538-544 (2006).

(i) Open Access This article is licensed under a Creative Commons Attribution 4.0 International License, which permits use, sharing, adaptation, distribution and reproduction in any medium or format, as long as you give appropriate credit to the original author(s) and the source, provide a link to the Creative Commons license, and indicate if changes were made. The images or other third party material in this article are included in the article's Creative Commons license, unless indicated otherwise in a credit line to the material. If material is not included in the article's Creative Commons license and your intended use is not permitted by statutory regulation or exceeds the permitted use, you will need to obtain permission directly from the copyright holder. To view a copy of this license, visit http://creativecommons. org/licenses/by/4.0/.

(c) The Author(s) 2019 